\title{
Regulation of seizure spreading by neuroserpin and tissue-type plasminogen activator is plasminogen-independent
}

\author{
Manuel Yepes, ${ }^{1,2}$ Maria Sandkvist,${ }^{3}$ Timothy A. Coleman, ${ }^{4}$ Elizabeth Moore, ${ }^{1}$ \\ Jiang-Young Wu, ${ }^{5}$ David Mitola, ${ }^{6}$ Thomas H. Bugge, ${ }^{6}$ and Daniel A. Lawrence ${ }^{1}$ \\ ${ }^{1}$ Department of Vascular Biology, American Red Cross Holland Laboratory, Rockville, Maryland, USA \\ ${ }^{2}$ Department of Neurology, Georgetown University Hospital, Washington, DC, USA \\ ${ }^{3}$ Department of Biochemistry, American Red Cross Holland Laboratory, Rockville, Maryland, USA \\ ${ }^{4}$ Department of Protein Development, Human Genome Sciences Inc., Rockville, Maryland, USA \\ ${ }^{5}$ Department of Physiology and Biophysics, Georgetown School of Medicine, Washington, DC, USA \\ ${ }^{6}$ Proteases and Tissue Remodeling Unit, Oral and Pharyngeal Cancer Branch, National Institute of Dental \\ and Craniofacial Research, NIH, Bethesda, Maryland, USA
}

Address correspondence to: Daniel A. Lawrence, Vascular Biology Department, Holland Laboratory, American Red Cross, 15601 Crabbs Branch Way, Rockville, Maryland 20855, USA.

Phone: (301) 517-0356; Fax: (301) 738-0794; E-mail: Lawrenced@usa.redcross.org.

Received for publication September 27, 2001, and accepted in revised form April 30, 2002.

Tissue-type plasminogen activator (tPA) is a highly specific serine proteinase expressed in the CNS during events that require neuronal plasticity. In this study we demonstrate that endogenous tPA mediates the progression of kainic acid-induced (KA-induced) seizures by promoting the synchronization of neuronal activity required for seizure spreading, and that, unlike KA-induced cell death, this activity is plasminogen-independent. Specifically, seizure induction by KA injection into the amygdala induces tPA activity and cell death in both hippocampi, and unilateral treatment of rats with neuroserpin, a natural inhibitor of tPA in the brain, enhances neuronal survival in both hippocampi. Inhibition of tPA within the hippocampus by neuroserpin treatment does not prevent seizure onset but instead markedly delays the progression of seizure activity in both rats and wild-type mice. In tPA-deficient mice, seizure progression is significantly delayed, and neuroserpin treatment does not further delay seizure spreading. In contrast, plasminogen-deficient mice show a pattern of seizure spreading and a response to neuroserpin that is similar to that of wild-type animals. These findings indicate that tPA acts on a substrate other than plasminogen and that the effects of neuroserpin on seizure progression and neuronal cell survival are mediated through the inhibition of tPA.

J. Clin. Invest. 109:1571-1578 (2002). doi:10.1172/JCI200214308.

\section{Introduction}

A remarkable feature of the CNS is its ability to undergo rapid activity-dependent remodeling of neuronal connections in a process that is referred to as synaptic plasticity. This process appears to depend, at least in part, on the activity of specific proteinases, and on the balance between proteinase activity and inhibition. It is thought that controlled local proteolytic activity mediates the assembly and disassembly of different components needed for axonal path-finding, and for the establishment of new neuronal connections.

Tissue-type plasminogen activator (tPA) is expressed at low levels within the CNS, but its synthesis is increased by events that require synaptic plasticity, such as long-term potentiation, kindling, seizures, and motor learning (1-3). The primary substrate for tPA in vivo is the zymogen plasminogen, which tPA activates to the broad-specificity proteinase plasmin. Outside the CNS, tPA is primarily a thrombolytic enzyme, since plasmin's principal substrate is fibrin (4). However, within the CNS the roles of tPA and plasmin are not well characterized, and their primary substrates are not known. For example, both tPA-deficient $\left(t \mathrm{PA}^{-/}\right)$and plasminogen-deficient $\left(\mathrm{Plg}^{-/-}\right)$mice have been shown to be resistant to direct kainic acid-induced (KA-induced) neuronal death $(5,6)$, suggesting that $\mathrm{PA}$ acts through the activation of plasmin. However, while $t P A^{-/-}$mice show reduced seizure-dependent mossy fiber outgrowth, $\mathrm{Plg}^{-1-}$ mice do not (7), suggesting that plasmin may not be the primary substrate for tPA within the $\mathrm{CNS}$. Likewise, $t P A^{-/-}$mice show a decrease in infarct volume and an increase in neuronal survival compared with wild-type animals following middle cerebral artery occlusion (8), while animals deficient in plasminogen show an increase in infarct volume, suggesting a plasminogen-independent function for tPA in cerebral ischemia (9). However, the precise roles of tPA and plasminogen in these events are unclear. 
Neuroserpin is a recently identified member of the serine proteinase inhibitor (serpin) gene family (10); it efficiently inhibits tPA and is expressed primarily in neurons in areas of the brain where tPA has been previously identified $(11,12)$. Neuroserpin has been shown to be neuroprotective after ischemic stroke $(13,14)$, while mutations in the neuroserpin gene are associated with an autosomal-dominant dementia and a form of progressive myoclonic epilepsy (15-18). The association of neuroserpin mutations with myoclonic epilepsy, and the observations that $t P A^{-/-}$mice have a higher threshold for the onset of seizures after intraperitoneal delivery of KA or Metrazol (5), suggest that tPA and neuroserpin may play an important role in synaptic remodeling. Consistent with this hypothesis, electron microscopy analysis of ex vivo hippocampal sections has indicated that tPA activity is important for the rapid formation of perforated synapses (19). However, whether this activity occurs through the activation of plasmin was not shown. In the present study we examine the role of tPA, plasminogen, and neuroserpin in seizure progression and the resultant seizure-induced neuronal cell death. Our data suggest that the main action of tPA after the injection of an excitotoxin is to promote the propagation of seizures throughout the brain. Moreover, the action of tPA in the spreading of KA-induced abnormal electrical activity is a plasminogen-independent event that can be blocked by the administration of neuroserpin.

\section{Methods}

Animal preparation and surgery. We used adult SpragueDawley rats weighting 350-400 g, and C57BL $/ 6 \mathrm{~J}-\mathrm{Plg}^{-1-}$, C57BL/6J-tPA ${ }^{-/-}$, and C57BL/6J-PAI-1-1- mice or their wild-type and heterozygous littermates genotyped as described (20). Three microliters of $1.0 \mathrm{nM} \mathrm{KA}$ (A.G. Scientific, San Diego, California, USA) in PBS was injected unilaterally into the amygdalae of anesthetized animals using a 26-gauge Hamilton syringe (Harvard Apparatus, Holliston, Massachusetts, USA). The coordinates of the injection of KA for rats were: bregma, $-3.60 \mathrm{~mm}$; medial-lateral, $5 \mathrm{~mm}$; and dorsoventral, $9 \mathrm{~mm}$. For mice they were: bregma, $-1.58 \mathrm{~mm}$; medial-lateral, $3.3 \mathrm{~mm}$; and dorso-ventral, $4.5 \mathrm{~mm}$. PBS or $16 \mu \mathrm{M}$ recombinant neuroserpin in PBS, purified as described (11) and containing less than 1 endotoxin unit/mg, was sterile-filtered and injected at these coordinates: bregma, $-3.60 \mathrm{~mm}$; medial-lateral, 2.5 $\mathrm{mm}$; and dorso-ventral $3.5 \mathrm{~mm}$ (rats); and bregma, $-1.70 \mathrm{~mm}$; medial-lateral, $1.5 \mathrm{~mm}$; and dorso-ventral, $2 \mathrm{~mm}$ (mice) $(21,22)$. Surgical incision through the corpus callosum was performed as described (23), and, after 1 hour, rats were euthanized and brain extracts prepared for SDS-PAGE zymography.

In situ proteinase activity, neuroserpin detection, and zymography. Rats were perfused 10,30, and 60 minutes after injection of KA. The brains were then removed, immediately frozen in OCT, and stored at $-70^{\circ} \mathrm{C}$. Cryostat sections $(5 \mu \mathrm{m})$ were stained with $100 \mu \mathrm{M}$
4',6-diamidino-2-phenylindole-2HCl (DAPI) for 30 seconds, rinsed in PBS, and then analyzed for in situ proteinase activity as described (24), with modifications for tPA (13). Briefly, 100- $\mu$ l overlays of $1 \%$ agarose in PBS containing $1 \mu \mathrm{g} / \mathrm{ml}$ BODIPY TR-X casein (Molecular Probes Inc., Eugene, Oregon, USA), $5 \mathrm{mM}$ EDTA with or without plasminogen (Molecular Innovations Inc., Southfield, Michigan, USA), with or without anti-tPA antibodies were applied to prewarmed tissue and sealed under glass coverslips. The slides were incubated for 2 hours at $37^{\circ} \mathrm{C}$ and then analyzed for casein fluorescence as described (24). For neuroserpin antigen detection, adjacent sections were analyzed, and slides were washed twice with PBS between steps. Sections were fixed in $0.3 \%$ hydrogen peroxide in methanol for 30 minutes, stained for 30 seconds with DAPI, blocked for 30 minutes in $0.1 \%$ $\mathrm{BSA} / 10 \%$ goat serum, and incubated with rabbit antineuroserpin (1:1000) for 30 minutes, followed by goat anti-rabbit Alexa Fluor 488 (Molecular Probes Inc.) (1:500) for 30 minutes. For SDS-PAGE zymography, a region of the brain containing the dorsal hippocampus from neuroserpin- and PBS-treated animals was dissected from both hemispheres 1 hour after the injection of KA, frozen in dry ice, and stored at $-70^{\circ} \mathrm{C}$. Protein extraction and plasminogen-dependent casein agarose zymography were performed as described (13). Hippocampal cell count. For quantification of seizureinduced cell loss in rats after the injection of either PBS or neuroserpin, the rats were perfused by intracardiac puncture with PBS followed by $10 \%$ buffered formalin, and the brains were postfixed for 24 hours in $70 \%$ ethanol. Serial sections $(5 \mu \mathrm{m})$ through the dorsal hippocampus were prepared and stained with hematoxylin and eosin (H\&E). Each section was observed microscopically and subjected to quantitative analysis by classifying cells as alive or dead based on their appearance in $\mathrm{H} \& \mathrm{E}$. The average of each category from different hippocampal regions was compared with similar measurements obtained in sham-treated rats.

Seizure classification and hippocampal local field potential recording. Animals were observed for 120 minutes, and clinical seizure behavior was classified as follows: 1 , myoclonic jerks involving the head and neck; 2 , unilateral tonic-clonic activity in the limbs; 3 , generalized bilateral tonic-clonic activity. Electroencephalogram (EEG) was recorded for 15 minutes before injection of KA and PBS or neuroserpin and for 2 hours thereafter using local field potential electrodes placed in the CA1 region of the hippocampus. A reference electrode was placed in the cerebellum. With this monopolar recording arrangement, the EEG signal primarily records neuronal activity within the hippocampal tissue (hippocampal seizure activity).

Statistical analysis. All data are presented as mean \pm SE and were analyzed for significance using a Student's $t$ test. $P$ values less than 0.05 were considered statistically significant and are indicated in the figures by an asterisk. 


\section{Results}

Animal model of seizures. To study the role of tPA in KAinduced seizure progression and seizure-induced neuronal death within the hippocampus, it is important to use a model in which the hippocampal cells are not in direct contact with KA. In contrast to other studies (5) where cell death was caused by the direct action of $\mathrm{KA}$ in the hippocampus, in the present study the excitotoxin was injected into the amygdala, a brain structure that is part of the limbic system but that is distant from the hippocampus. Analysis of brains injected with ${ }^{3} \mathrm{H}-\mathrm{KA}$ at a concentration 35 -fold higher than those used in the present study indicated that less than $2 \%$ of the ${ }^{3} \mathrm{H}-\mathrm{KA}$ reached the ipsilateral hippocampus even 1 hour after injection (not shown). Thus, any effects observed in the hippocampal cell layers are the consequence of the seizure activity and not of the KA itself. Figure 1 shows a diagram of the model used.

Seizures induce tPA activity and neuroserpin antigen throughout the limbic system. Previous studies have indicated that experimentally induced seizures increase tPA gene expression throughout the brain (2), and tPA is thought to mediate excitotoxin-induced cell death (5). Likewise, tPA activity is increased in models of ischemic stroke, and neuroserpin treatment or overexpression has been shown to reduce neuronal cell death in these models $(13,14)$. Therefore, we examined the effect of experimentally induced seizures on tPA activity and neuroserpin antigen within the limbic system by in situ zymography and immunofluorescence (Figure 2 ). Both tPA activity and neuroserpin antigen were detected in the ipsilateral amygdala within 10 minutes of KA injection (Figure 2a). By 30 minutes after KA injection, both tPA and neuroserpin appeared to increase within the ipsilateral amygdala (Figure 2d) and were also now detected within the ipsilateral hippocampus (Figure 2e). By 60 minutes after KA injection, both tPA activity and neuroserpin antigen were clearly evident in the contralateral hippocampus (Figure $2 \mathrm{i}$ ). The proteolytic activity was due to tPA, since no activity was observed in the absence of plasminogen in the overlay gel (not shown), and since the inclusion of anti-tPA antibodies in the zymography completely blocked the activity (Figure $2, \mathrm{j}-1$, top panels). These results suggest that the injection of KA into the amygdala induces a progressive increase in the activity of tPA throughout the limbic system. The simultaneous increase of neuroserpin antigen in the same structures suggests a role for neuroserpin in regulating the action of tPA during seizure progression.

Neuroserpin blocks excitotoxin-induced cell death. The increase in neuroserpin levels observed during seizure spreading suggests that neuroserpin may play an important role in cell survival following seizures. To test this hypothesis, KA was injected into the amygdalae of rats, immediately followed by the administration of either PBS or neuroserpin into the ipsilateral hippocampus. Twenty-four hours later, viable cell counts were performed in the dorsal hippocampus.
The results of these experiments showed that hippocampal neuronal loss after KA-induced seizures occurred bilaterally (Figure 3 ) and that neuroserpin treatment appeared to reduce cell death in both hippocampi. This effect was highly significant in the CA3 layer of both hippocampi even though neuroserpin was injected only into the ipsilateral hippocampus.

TPA mediates spreading of seizure activity throughout the brain. In addition to blocking neuronal cell death, neuroserpin treatment also appeared to attenuate the progression and generalization of the convulsive activity. To further examine this unexpected finding, the sequence of convulsive behavior was analyzed in rats following the injection of KA into the amygdala and the administration of either PBS or neuroserpin into the hippocampus. The response to KA in PBS-treated animals was characterized first by unilateral tonicclonic contractions of the face followed by the forelimb, and subsequently by generalization involving all four limbs (Figure 4). The injection of neuroserpin into the hippocampus was not associated with a significant delay in the onset of ictal activity in the face, indicating that neuroserpin does not inhibit the action of KA directly. However, neuroserpin treatment significantly attenuated the spread of ictal activity to the forelimb by approximately 30 minutes $(P<0.05)$ and completely blocked generalization to bilateral contractions $(P<0.004)$. Treatment with inactive neuroserpin, cleaved in its reactive center loop (13), did not attenuate seizure progression, indicating that neuroserpin inhibitory activity is necessary for this effect (not shown). Since neuroserpin is a natural inhibitor of tPA in the brain, these observations suggest that tPA does not mediate the induction of seizure activity produced by the injection of KA into the amygdala but rather promotes the spreading and maintenance of the abnormal electrical activity.

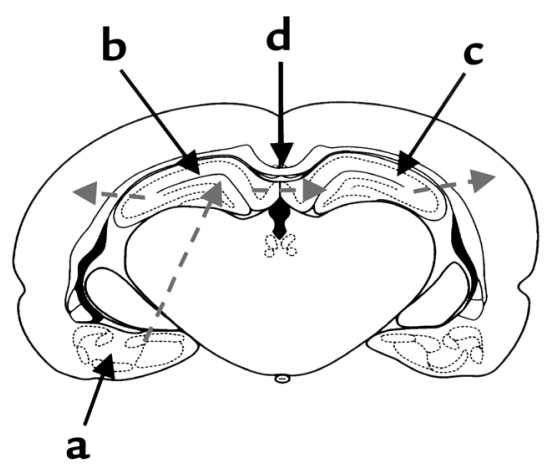

\section{Figure 1}

Model of KA-induced seizures in the limbic system. A diagram of a coronal section through the rat hippocampi and amygdaloid nuclei is shown. KA was injected into the amygdaloid nuclei (a). Dashed arrows show the propagation of the abnormal electrical activity, originating in the amygdala, first to the ipsilateral (b) and then to the contralateral (c) hippocampus through the corpus callosum (d). Neuroserpin or PBS was injected into the hippocampus ipsilateral to the amygdala injected with $\mathrm{KA}(\mathbf{b})$. 


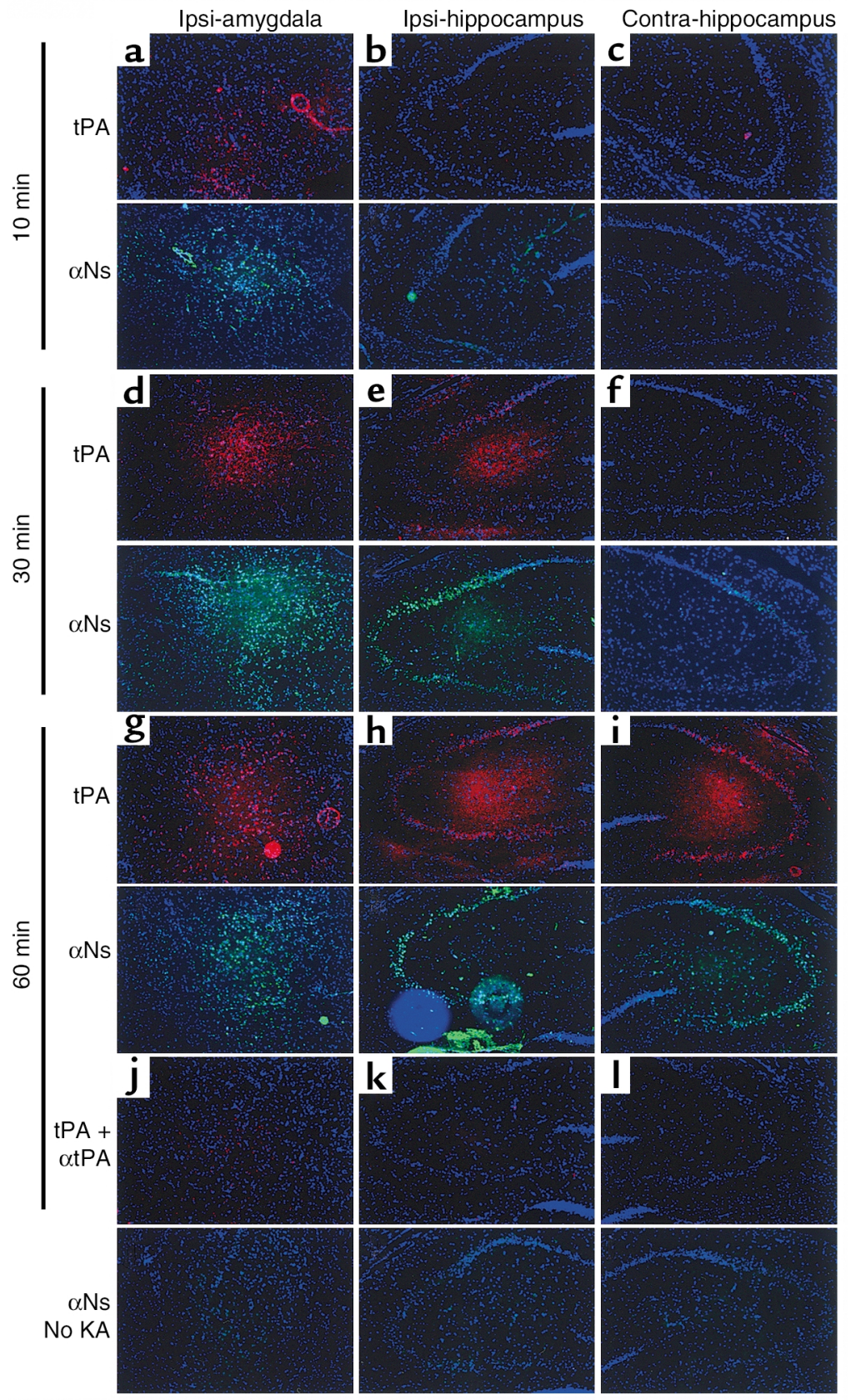

\section{Figure 2}

Kinetic analysis of tPA activity and neuroserpin antigen in the limbic system during seizure. Seizures were induced by KA injection into the amygdala as described in Methods, and the animals were euthanized at 10, 30, and 60 minutes after injection. Cryostat sections were stained with DAPI to visualize nuclei and then analyzed either for in situ tPA activity by plasminogen-casein zymography with internally quenched fluorescent casein (red) or for neuroserpin antigen by immunofluorescence (green). The top panel of each part shows tPA activity, and the bottom panel of each part shows neuroserpin antigen $(\alpha \mathrm{Ns})$. (a-c) Ten minutes after KA injection; (d-f) 30 minutes after injection; (g-i) 60 minutes after injection. (j-I) Top panels: 60 minutes after KA injection, with anti-tPA antibodies ( $\alpha \mathrm{tPA})$ in the overlay; bottom panels: normal rat brain with no KA injection. $\mathbf{a}, \mathbf{d}, \mathbf{g}$, and $\mathbf{j}$ show the basolateral amygdala ipsilateral (Ipsi) to the injection site; $\mathbf{b}, \mathbf{e}$, $\mathbf{h}$, and $\mathbf{k}$ show the ipsilateral hippocampus; $\mathbf{c}, \mathbf{f}, \mathbf{i}$, and I show the contralateral (Contra) hippocampus. Original magnification, $\times 100$.

These data suggest that the spreading and synchronization of ictal activity is a tPA-dependent event that is blocked by the administration of neuroserpin.

Neuroserpin blocks seizure-induced tPA activity. To examine the relative increase in tPA activity following seizures, semiquantitative SDS-PAGE zymography was performed on hippocampal extracts. This analysis demonstrated that there was a significant bilateral increase in tPA activity after the induction of seizures (Figure 6 , a and $b$ ), and that administration of neuroserpin into the ipsilateral hippocampus significantly decreased this activity in both hippocampi (Figure $6 \mathrm{~b})$. The decrease in tPA activity ipsilateral to the injection site was most likely the result of direct inhibition of tPA by neuroserpin.

Neuroserpin blocks electrographic spreading of ictal activity. The hippocampus has been implicated in the genesis and propagation of ictal activity originating in distant brain structures such as the amygdala. We therefore examined whether neuroserpin treatment also blocked the propagation of abnormal electrical activity associated with the injection of KA into the amygdala. Bilateral monitoring of hippocampal local field potentials demonstrated that neuroserpin treatment decreased the magnitude of interictal activity ipsilateral to the KA injection and blocked the development of hippocampal seizures. In contrast, PBS-treated animals demonstrated interictal activity ipsilateral to the KA injection that rapidly progressed to repeated episodes of bilateral hippocampal seizures (Figure 5, a and b; large black arrows).
To exclude the possibility that the inhibition of tPA activity in the contralateral hemisphere was due to neuroserpin diffusion across the midline, fluorescently labeled neuroserpin was injected into the ipsilateral hippocampus. One hour after injection, there was no detectable passage of the labeled protein to the contralateral hippocampus (data not shown). Thus, the reduction in tPA activity in the contralateral hippocampus must have been due to the blockade of seizure spreading. This suggests that the bilateral increase in tPA activity is a direct consequence of the spreading of the abnormal electrical activity generated by the injection of KA into the amygdala.

To test this hypothesis, the corpus callosum (Figure 1d), which is a structure containing the axonal 

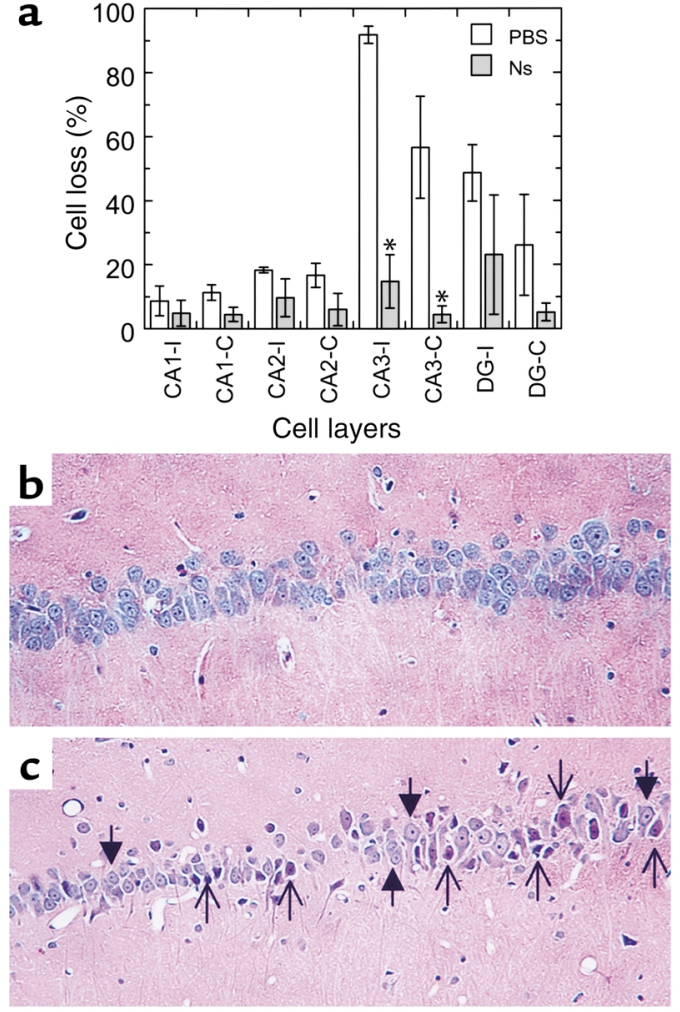

Figure 3

Quantitative analysis of neuronal loss in the hippocampus 24 hours after seizure. KA and neuroserpin treatments were as described in Methods. The animals were euthanized 24 hours after KA injection, tissue sections were stained with $\mathrm{H} \& \mathrm{E}$, and surviving neurons in the CA1-CA3 and dentate gyrus (DG) layers of the hippocampus were counted microscopically and compared with control sections from untreated animals. (a) The results of this analysis $(n=3$ for each condition). ${ }^{*} P<0.05$ relative to the same cell layer from PBS-treated animals. I, ipsilateral; C, contralateral. (b) An H\&E stain of a normal rat hippocampus at the junction of the CA2 and CA3 layers. (c) The same region 24 hours after $\mathrm{KA}$ injection. Original magnification, $\times 200$. The open arrows in $\mathbf{c}$ show examples of cells counted as dead, and the filled arrows show examples of cells counted as alive.

connections between the two hemispheres and is necessary for the propagation of seizure activity to the contralateral hemisphere, was surgically severed (callosotomy). If the increased tPA activity observed in the contralateral hippocampus is a direct consequence of the spreading of ictal activity through the midline, then this surgical procedure should prevent the increase in tPA activity in the contralateral hemisphere. SDSPAGE zymography of extracts following callosotomy and KA injection into the amygdala demonstrated an increase in tPA activity in the ipsilateral hippocampus, similar to that observed in control animals. However, in the contralateral hippocampus, there was no increase in tPA activity associated with seizures (Figure 6 , a and $\mathrm{b} ; P<0.02$ ). These data indicate that both the rise in tPA activity and the spreading of seizure activity to the contralateral hemisphere resulted from direct stimulation of the ipsilateral amygdala, and that the clinical and electroencephalographic inhibition of seizure spreading observed with neuroserpin treatment is very probably due to inhibition of tPA activity in the ipsilateral hippocampus.

Analysis of seizure spreading in $t P A$-and plasminogen-deficient mice. To directly confirm the role of tPA activity in seizure spreading, $t \mathrm{PA}^{-/}$and congenic wild-type mice were examined after the injection of KA into the amygdala. These results demonstrated that, as in rats, there is a rapid progression of seizure behavior from the face to the limb, and finally to the four extremities (generalization) in wild-type mice treated with PBS (Figure 7). Also, as in rats, neuroserpin-treated wild-type mice showed a significant reduction in the progression of convulsive activity. In contrast, $t \mathrm{PA}^{-/-}$mice showed a marked decrease in the rate of seizure progression from the face to the limbs compared with wild-type mice $(P \leq 0.02)$, and treatment with neuroserpin did not further prolong this time (Figure 7). Moreover, none of the $t P A^{-/-}$mice generalized to bilateral seizure activity. Together, these results confirm our hypothesis that tPA appears to promote the spreading of the abnormal electrical activity throughout the brain. Furthermore, these results demonstrate that neuroserpin inhibits seizure progression in a tPA-dependent manner, suggesting that the primary function of neuroserpin is the inhibition of tPA activity within the CNS. Consistent with this interpretation, the spreading of seizure activity in $\mathrm{PAI}-1^{-/}$mice was indistinguishable from that observed in wild-type animals, suggesting that PAI-1 is not an important regulator of tPA in this model (Figure 7). Finally, to determine whether tPA was acting through the generation of plasmin, we recorded the progression of seizure behavior in $\mathrm{Plg}^{-/-}$mice treated with PBS or neuroserpin. Unlike the $\mathrm{PA}^{-/-}$mice, the spreading of seizure activity in the $\mathrm{Plg}^{-1}$ mice was essentially identical to that observed in wild-type animals, and also, like in wild-type animals, treatment with neuroserpin significantly delayed the generalization of the seizure activity. This demonstrates that tPA promotes the spreading of ictal activity throughout the brain in a plasminogenindependent manner.

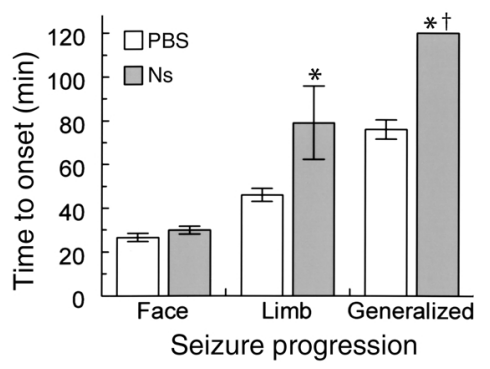

Figure 4

Clinical analysis of seizure behavior in rats. KA-treated rats were injected with neuroserpin (Ns) or PBS as described in Methods, and seizure behavior was recorded for 2 hours. Convulsive behavior was classified in three categories: 1 , seizures affecting one side of the face or neck; 2, convulsion involving limbs only on one side; and 3, generalized bilateral seizure activity involving four limbs. For each condition, $n=6 .{ }^{*} P<0.05$ relative to PBS-treated animals. ${ }^{+N o n e}$ of the animals generalized during the experiment. 
$\mathbf{a}$

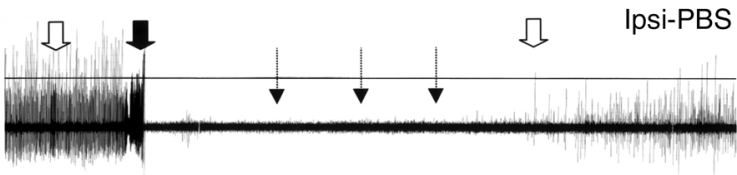

b

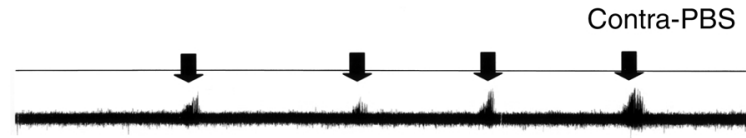

c

Ipsi-Ns

d

Contra-Ns

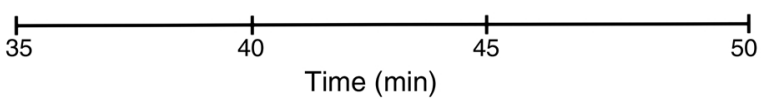

\section{Discussion}

The limbic system is a circuit that interconnects several structures within the brain, including the amygdala and the hippocampus. Injection of the glutamate analog KA into the amygdala induces the propagation of aberrant electrical activity along the limbic circuit, leading to neuronal death within distant vulnerable targets such as the hippocampus (25-28). This model permits analysis of the propagation of seizures along the limbic circuit and assures that any changes observed within the hippocampi, such as neuronal death or increased tPA activity, are a consequence of the propagation of the seizure activity generated in the amygdala and are not due to the direct action of KA on hippocampal cells.

Our results indicate that the levels of both neuroserpin antigen and tPA activity increase very early after seizures, especially within the CA2-CA3 layers in

\section{Figure 6}

Analysis of tPA activity in brain extracts. (a) SDS-PAGE zymography of brain extracts. Lane 1 is a mixture of human tPA and a rat kidney extract as a marker for rat urokinase plasminogen activator (UPA). Lane 2 is empty, and lanes 3-10 are hippocampal extracts 1 hour after KA injection. Lanes 3, 5, 7, and 9 are ipsilateral (I) and lanes 4, 6,8 , and 10 are contralateral (C) to the injection. Lanes 3 and 4 are from sham-operated animals that did not receive KA (Sham), and all other lanes show animals that received KA. Lanes 5 and 6 show PBStreated animals, lanes 7 and 8 are from neuroserpin-treated animals (Ns), and lanes 9 and 10 are from animals that underwent corpus callosotomy (Call). (b) Quantitative analysis of PA activity from SDSPAGE zymography of brain extracts 1 hour after KA injection. The results represent the average fold increase in TPA activity in the hippocampus ipsilateral (Ipsi) and contralateral (Contra) to the KA injection, relative to normal base-line tPA activity in sham-operated animals. PBS and Ns represent animals treated with PBS or neuroserpin, respectively ( $n=5$ for each condition), and Call represents brain extracts of animals that underwent corpus callosotomy $(n=2)$. ${ }^{*} P<0.05$ relative to PBS-treated animals. AU, arbitrary units.

\section{Figure 5}

EEG recording in rats with KA-induced seizures. Monopolar electrodes were positioned into the ipsilateral or contralateral hippocampus as described in Methods. $\mathbf{a}$ and $\mathbf{c}$ are ipsilateral (Ipsi) and $\mathbf{b}$ and $\mathbf{d}$ are contralateral (Contra) to the KA injection. (a and $\mathbf{b}$ ) PBStreated animals; (c and d) neuroserpin-treated ( Ns) animals. In $\mathbf{a}$ and b, the large black arrows indicate ictal activity (seizure), the large white arrows indicate interictal activity, and the dashed black arrows indicate the depression of the electrical activity immediately following seizure. In c, interictal activity is observed throughout the entire trace, and in $\mathbf{d}$, no interictal activity is seen. Animals were monitored for 120 minutes ( $n=3$ for each condition).

both hippocampi. We hypothesize that the progressive rise in tPA activity observed after the injection of $\mathrm{KA}$ is a direct result of the propagation of the seizure activity along the limbic system. This conjecture is supported by the observations that (a) large reductions in tPA activity are seen in both hippocampi upon the injection of neuroserpin into only the ipsilateral hippocampus, and (b) tPA activity does not rise in the contralateral hippocampus when communication between the two hemispheres is surgically interrupted. These data contrast with models of intrahippocampal injection of KA where tPA antigen was shown to increase within the CA1 layer by 5 hours after KA injection (29). These data suggest that seizures increase tPA activity by a different mechanism than does the intrahippocampal injection of KA. Since neuronal expression of tPA is known to rapidly increase after events that involve neuronal stimulation (3), it is likely that the very early rise in tPA activity that we observed during seizures stems from the release of tPA from neurons. In contrast, the late increase in tPA expression associated with the intrahippocampal injection of KA seems to originate from activated microglial cells, which are known to

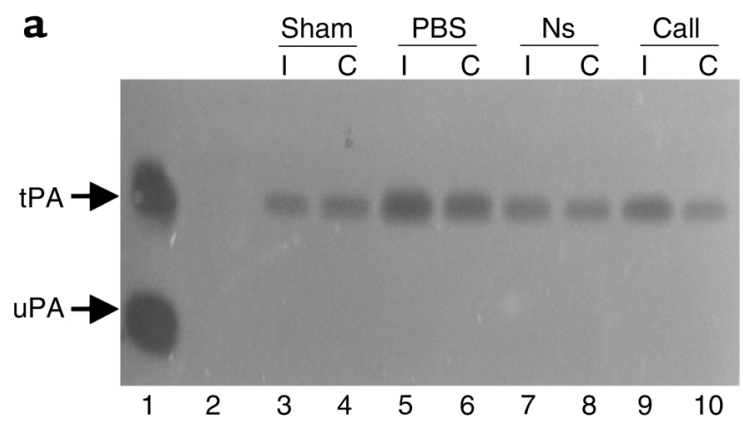

b

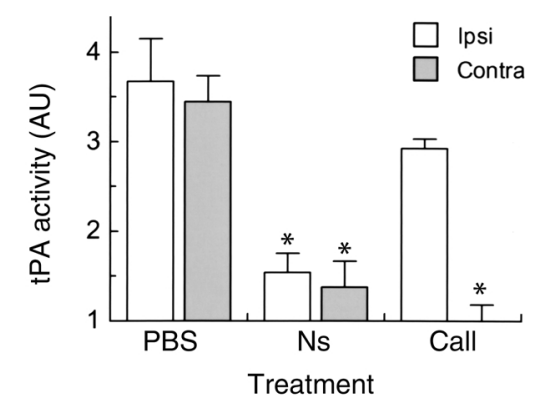




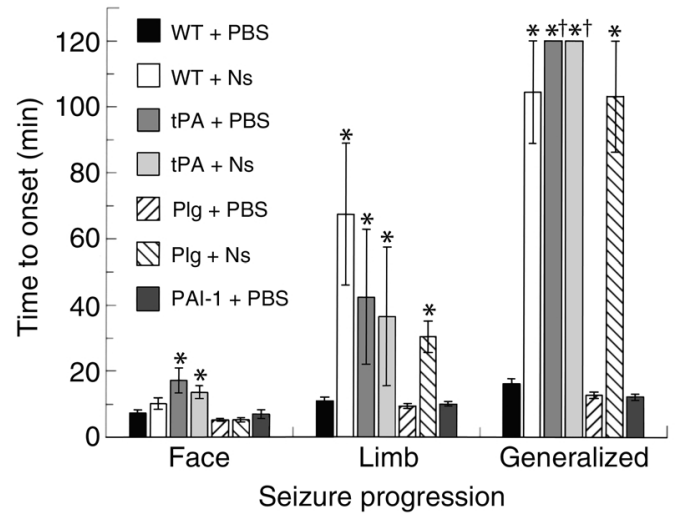

Figure 7

Clinical analysis of seizure behavior in mice. KA-treated mice were injected with neuroserpin (Ns) or PBS as described in Methods, and seizure behavior was recorded for 2 hours. Convulsive behavior was classified as in Figure 4. WT, wild-type mice; tPA, $t P A^{-/-}$mice; Plg, $\mathrm{Plg}^{-1-}$ mice; PAI-1, PAl-1-1- mice. ${ }^{*} P<0.05$ relative to PBS-treated wild-type animals. ${ }^{+N}$ one of the animals generalized at this time. For each condition, $n=5-10$.

express significant levels of plasminogen-dependent proteolytic activity and to increase in number $24-48$ hours after KA injection (30).

The finding of a large increase in tPA activity very early after seizures in both hippocampi, in the CA3 layer in particular, is noteworthy since this is the same area where the most significant cell loss occurred 24 hours after the induction of seizures, and where the most significant effect of neuroserpin treatment on cell survival was observed. This suggests not only that tPA mediates seizure-induced cell death, but also that neuroserpin promotes neuronal survival, most likely by inhibition of tPA activity. Furthermore, we can speculate that the increased detection of neuroserpin in the hippocampus following seizures may represent an endogenous neuroprotective response to increased tPA levels, and that neuroserpin may act as a natural neuronal survival factor. However, when local tPA levels rise too high, the protective effect of endogenous neuroserpin is overwhelmed. This result is similar to previous studies indicating that neuroserpin enhances neuronal survival after stroke $(13,14)$. Finally, these data indicate that although KA-induced cell death and seizure-induced cell death are both mediated by tPA, they are caused by different mechanisms. The former is a local event caused by the direct action of the excitotoxin on the neurons, while the latter occurs in both hippocampi and results from the spread of the abnormal electrical activity throughout neuronal circuits.

It is clear from our results that inhibition of the rise in tPA activity, either biochemically with neuroserpin, physically by callosotomy, or genetically in tPA-deficient mice, significantly reduces the clinical spreading of seizure activity. Likewise, analysis of hippocampal local field potentials indicated that neuroserpin treatment blocked the development of seizures in both hippocampi. The cells of the CA3 area receive input from the dentate gyrus and not only send excitatory connections into the CA1 area but also send recurrent excitatory axons back into the CA3 area (31). This recurrent excitatory pathway is responsible for the ability of the CA3 area to fire interictal bursts. Our data indicate that neuroserpin administration diminished the generation of both interictal activity and subsequent electrographic seizures in both hippocampi. Since most of the tPA activity following the injection of KA is seen in the CA3 area, which is responsible for the generation of interictal bursts and subsequent generalization of seizure activity, we postulate that the clinical and electrographic effects observed upon the injection of neuroserpin are due to $\mathrm{PAA}$ inhibition.

The mechanism whereby tPA could promote the neuronal hypersynchrony needed for the generation of a seizure is unknown; however, based on these results we can speculate that the early release of tPA into the synaptic space during seizures may promote the progression and spreading of the abnormal electrical activity in the form of a positive feedback loop, either by strengthening existing synaptic contacts or by creating new ones. Consistent with this hypothesis is the rise in tPA activity observed in the contralateral hippocampus, which occurs within 60 minutes of KA injection and precedes the clinical observation of seizure generalization by at least 15 minutes. Likewise, the changes observed in the contralateral hippocampus after treatment with neuroserpin (i.e., decreased tPA activity, increased neuronal survival, and absence of electrographic seizures) are all most likely due to interruption of the seizure propagation by interfering with the formation of new synaptic contacts between both hippocampi. Previous studies have suggested that tPA is associated with morphological changes in vitro (32), and with the rapid establishment of activity-induced synaptic plasticity in vivo (3). Our results strongly support this hypothesis and suggest that tPA is needed for seizure propagation throughout the brain, perhaps by facilitating the rapid remodeling and reorganization of the synapse, or by fostering the creation of new synaptic contacts needed for the spreading and maintenance of the abnormal electrical activity.

One potential synaptic substrate for tPA activity is the NR1 subunit of the $\mathrm{N}$-methyl $\mathrm{D}$-aspartate (NMDA) receptor. The ability of NMDA to induce seizure-like phenomena and the inhibition of epileptic activity by NMDA receptor antagonists (33) suggests that this family of receptors is directly involved in the propagation of seizure activity. The finding that tPA can cleave the NR1 subunit of the NMDA receptor in vitro, enhancing the influx of calcium into the cell (34), supports the suggestion that NMDAinduced cell death might be mediated by tPA. Neuroserpin may block this proposed interaction with the NMDA receptor by inhibiting the action of tPA released in the synaptic space, thus decreasing the influx of calcium into the cell, which may dampen the 
propagation of ictal activity and promote subsequent neuronal survival. Finally, the results with $\mathrm{Plg}^{-/-}$mice indicate that, in the case of seizure spreading, the action of tPA is plasminogen-independent. This is in contrast to the direct neurotoxic effects of KA, since in studies where KA was injected directly into the hippocampus, both tPA and plasminogen deficiency were shown to be neuroprotective $(5,6)$. Thus, our data demonstrate a role for tPA in vivo that is plasminogen-independent and suggest that within the CNS there is an as-yet unidentified substrate(s) for tPA, possibly the NR1 subunit of the NMDA receptor (34).

\section{Acknowledgments}

This work was supported by NIH grants HL-55374 and HL-55747 to D.A. Lawrence, NS-36477 to J.-Y. Wu, and NS-02223 to M. Yepes. The authors wish to thank Dudley Strickland for helpful discussions and critical reading of the manuscript, and Elizabeth Smith for superb histological work.

1. Carroll, P.M., Tsirka, S.E., Richards, W.G., Frohman, M.A., and Strickland, S. 1994. The mouse tissue-plasminogen activator gene 5 ' flanking region directs appropriate expression in development and a seizureenhanced response in the CNS. Development. 120:3173-3183.

2. Qian, Z., Gilbert, M.E., Colicos, M.A., Kandel, E.R., and Kuhl, D. 1993 Tissue-plasminogen activator is induced as an immediate-early gene during seizure, kindling and long-term potentiation. Nature. 361:453-457.

3. Seeds, N.W., Williams, B.L., and Bickford, P.C. 1995. Tissue-plasminogen activator induction in Purkinje neurons after cerebellar motor learning. Science. 270:1992-1994.

4. Bugge, T.H., et al. 1996. Loss of fibrinogen rescues mice from the pleiotropic effects of plasminogen deficiency. Cell. 87:709-719.

5. Tsirka, S.E., Gualandris, A., Amaral, D.G., and Strickland, S. 1995. Excitotoxin-induced neuronal degeneration and seizure are mediated by tissue-plasminogen activator. Nature. 377:340-344.

6. Tsirka, S.E., Bugge, T.H., Degen, J.L., and Strickland, S. 1997. Neuronal death in the central nervous system demonstrates a non-fibrin substrate for plasmin. Proc. Natl. Acad. Sci. USA. 94:9779-9781.

7. Wu, Y.P., et al. 2000. The tissue-plasminogen activator (tPA)/plasmin extracellular proteolytic system regulates seizure-induced hippocampal mossy fiber outgrowth through a proteoglycan substrate. J. Cell Biol. 148:1295-1304.

8. Wang, Y.F., et al. 1998. Tissue-plasminogen activator (tPA) increases neuronal damage after focal cerebral ischemia in wild-type and tPA-deficient mice. Nat. Med. 4:228-231.

9. Nagai, N., De Mol, M., Lijnen, H.R., Carmeliet, P., and Collen, D. 1999. Role of plasminogen system components in focal cerebral ischemic infarction: a gene targeting and gene transfer study in mice. Circulation. 99:2440-2444.

10. Osterwalder, T., Contartese, J., Stoeckli, E.T., Kuhn, T.B., and Sonderegger, P. 1996. Neuroserpin, an axonally secreted serine protease inhibitor. EMBO J. 15:2944-2953.

11. Hastings, G.A., et al. 1997. Neuroserpin, a brain-associated inhibitor of tissue-plasminogen activator is localized primarily in neurons. Implica- tions for the regulation of motor learning and neuronal survival. J. Biol. Chem. 272:33062-33067.

12. Osterwalder, T., et al. 1998. The axonally secreted serine proteinase inhibitor, neuroserpin, inhibits plasminogen activators and plasmin but not thrombin. J. Biol. Chem. 273:2312-2321.

13. Yepes, M., et al. 2000. Neuroserpin reduces cerebral infarct volume and protects neurons from ischemia-induced apoptosis. Blood. 96:569-576.

14. Cinelli, P., et al. 2001. Neuroserpin, a neuroprotective factor in focal ischemic stroke. Mol. Cell. Neurosci. 18:443-457.

15. Davis, R.L., et al. 1999. Familial dementia caused by polymerization of mutant neuroserpin. Nature. 401:376-379.

16. Davis, R.L., et al. 1999. Familial encephalopathy with neuroserpin inclusion bodies. Am. J. Pathol. 155:1901-1913.

17. Takao, M., et al. 2000. Neuroserpin mutation S52R causes neuroserpin accumulation in neurons and is associated with progressive myoclonus epilepsy. J. Neuropathol. Exp. Neurol. 59:1070-1086.

18. Yazaki, M., et al. 2001. Biochemical characterization of a neuroserpin variant associated with hereditary dementia. Am. J. Pathol. 158:227-233.

19. Neuhoff, H., Roeper, J., and Schweizer, M. 1999. Activity-dependent formation of perforated synapses in cultured hippocampal neurons. Eur.J. Neurosci. 11:4241-4250.

20. Bugge, T.H., et al. 1996. Fatal embryonic bleeding events in mice lacking tissue factor, the cell-associated initiator of blood coagulation. Proc. Natl. Acad. Sci. USA. 93:6258-6263.

21. Paxinos, G., and Watson, C. 2001. The rat brain in stereotaxic coordinates Academic Press Inc. San Diego, California, USA. 1-78.

22. Franklin, K., and Paxinos, G. 2001. The mouse brain in stereotaxic coordinates. Academic Press Inc. San Diego, California, USA. 1-93.

23. Crowne, D.P., and Richardson, C.M. 1985. A method to section the corpus callosum in the rat. Physiol. Behav. 34:847-850.

24. Scott, M.E., Dossani, Z.Y., and Sandkvist, M. 2001. Directed polar secretion of protease from single cells of Vibrio cholerae via the type II secretion pathway. Proc. Natl. Acad. Sci. USA. 98:13978-13983.

25. Ben Ari, Y., Lagowska, J., Tremblay, E., and Le Gal, L.S. 1979. A new model of focal status epilepticus: intra-amygdaloid application of kainic acid elicits repetitive secondarily generalized convulsive seizures. Brain Res. 163:176-179.

26. Lothman, E.W., and Collins, R.C. 1981. Kainic acid induced limbic seizures: metabolic, behavioral, electroencephalographic and neuropathological correlates. Brain Res. 218:299-318.

27. Tuunanen, J., Lukasiuk, K., Halonen, T., and Pitkanen, A. 1999. Status epilepticus-induced neuronal damage in the rat amygdaloid complex: distribution, time-course and mechanisms. Neuroscience. 94:473-495.

28. Ben Ari, Y., Tremblay, E., Ottersen, O.P., and Meldrum, B.S. 1980. The role of epileptic activity in hippocampal and "remote" cerebral lesions induced by kainic acid. Brain Res. 191:79-97.

29. Salles, F.J., and Strickland, S. 2002. Localization and regulation of the tissue-plasminogen activator-plasmin system in the hippocampus. J. Neurosci. 22:2125-2134.

30. Andersson, P.B., Perry, V.H., and Gordon, S. 1991. The kinetics and morphological characteristics of the macrophage-microglial response to kainic acid-induced neuronal degeneration. Neuroscience. 42:201-214.

31. Swanson, T.H. 1996. The electrophysiology of the human hippocampus and temporal cortex. In The treatment of epilepsy: principles and practice. E. Wyllie, editor. Williams \& Wilkins. Baltimore, Maryland, USA. 43-52.

32. Neuman, T., Stephens, R.W., Salonen, E.M., Timmusk, T., and Vaheri, A. 1989. Induction of morphological differentiation of human neuroblastoma cells is accompanied by induction of tissue-type plasminogen activator. J. Neurosci. Res. 23:274-281.

33. Dingledine, R., McBain, C.J., and McNamara, J.O. 1990. Excitatory amino acid receptors in epilepsy. Trends Pharmacol. Sci. 11:334-338.

34. Nicole, O., et al. 2001. The proteolytic activity of tissue-plasminogen activator enhances NMDA receptor-mediated signaling. Nat. Med. 7:59-64. 